\title{
Numerical Solutions of Fifth and Sixth Order Nonlinear Boundary Value Problems by Daftardar Jafari Method
}

\author{
Inayat Ullah, Hamid Khan, and M. T. Rahim \\ Department of Mathematics, National University of Computer and Emerging Sciences, FAST Peshawar Campus, \\ Peshawar 25000, Pakistan \\ Correspondence should be addressed to Inayat Ullah; p119952@nu.edu.pk
}

Received 5 November 2013; Revised 8 February 2014; Accepted 13 February 2014; Published 30 March 2014

Academic Editor: Fu-Yun Zhao

Copyright (C) 2014 Inayat Ullah et al. This is an open access article distributed under the Creative Commons Attribution License, which permits unrestricted use, distribution, and reproduction in any medium, provided the original work is properly cited.

Fifth and sixth order boundary value problems are solved using Daftardar Jafari method (DJM). DJM is introduced by DaftardarGejji and Jafari (2006). The approach provides the solution in the form of a rapidly convergent series. The comparison among Daftardar Jafari method (DJM), Adomian decomposition method (ADM), homotopy perturbation method (HPM), variation iteration method (VIM), and the iterative method (ITM) are displayed in table form which shows the efficiency of DJM for the solution of fifth and sixth order BVPs.

\section{Introduction}

Fifth order boundary value problems arise in the mathematical modeling of viscoelastic flows $[1,2]$. Agarwal, in [3], presents the conditions for the uniqueness and existence of the solutions of such type of problems. In [4], Khan investigated the solution of the fifth order boundary value problem using the finite-difference method. The improvement produced when the sixth-degree B-spline functions were used in [5]. Wazwaz, in [6], presented the numerical solution of fifth order BVP using the Adomian decomposition method and its modified form.

Sixth order boundary value problems arise in astrophysics. Modeling by sixth order BVPs occurs when the narrow convecting layers bounded by stable layers which are believed to surround A-type stars. To solve such BVPs, nonnumerical techniques were developed by Twizell and Boutayeb [7] and Baldwin [8]. Baldwin also considers the same work in his book in [9]. Chawla and Katti, in [10], introduced the numerical methods for the solution of higher order differential equations. Wazwaz, in [11], used the Adomian decomposition method and its modified form to solve such problems.

The aim of this research paper is to solve fifth and sixth order boundary value problems by Daftardar Jafari method (DJM). Recently Daftardar-Gejji and Bhalekar used this method to solve fractional boundary value problems with Dirichlet boundary conditions [12]. Ullah et al. [13] used DJM to solve higher order boundary value problems and got excellent results. DJM is a computer friendly iterative method to solve, especially, nonlinear BVPs. In the Adomian decomposition method [6] one has to compute Adomian polynomials which involve tedious calculations, whereas, in DJM, a couple of computer commands are sufficient to deal nonlinear BVPs. Results obtained from DJM are in higher agreement than the other numerical solutions such as ADM, ITM, VIM, and HPM.

In the first section, the basic idea of DJM is introduced. Comparison between ADM and DJM and convergence of DJM and its analysis are discussed in the respective sections. The last section contains the numerical solutions of fifth and sixth order nonlinear boundary value problems. Tables 1,2 , and 3 are constructed in order to compare the results obtained.

\section{Basic Idea of Daftardar Jafari Method}

Let us consider the nonlinear functional equation [12]

$$
u=f+L(u)+N(u),
$$


TABLE 1: Fifth order nonlinear boundary value problem (Example 1).

\begin{tabular}{lccccc}
\hline$x$ & Analytic solution & Error (ADM) & Error (HPM) & Error (VIM) & Error (DJM) \\
\hline 0.0 & 1.000000000 & 0.0000 & 0.0000 & 0.0000 & $1.0 E-9$ \\
0.1 & 1.105170918 & $1.0 E-9$ & $1.0 E-9$ & $2.0 E-9$ & $7.9 E-15$ \\
0.2 & 1.221402758 & $2.0 E-9$ & $2.0 E-9$ & $1.0 E-8$ & $2.0 E-8$ \\
0.3 & 1.349858808 & $1.0 E-8$ & $1.0 E-8$ & $3.1 E-8$ & $3.7 E-14$ \\
0.4 & 1.491824698 & $2.0 E-8$ & $2.0 E-8$ & $3.7 E-8$ & $5.15-13$ \\
0.5 & 1.648721271 & $3.1 E-8$ & $3.1 E-8$ & $4.1 E-8$ & $6.8 E-13$ \\
0.6 & 1.822118800 & $3.7 E-8$ & $3.7 E-8$ & $3.1 E-8$ & $7.5 E-13$ \\
0.7 & 2.013752707 & $4.1 E-8$ & $4.1 E-8$ & $1.4 E-8$ & $6.2 E-13$ \\
0.8 & 2.225540928 & $3.1 E-8$ & $3.1 E-8$ & 0.0000 & $3.1 E-13$ \\
0.9 & 2.459603111 & $1.4 E-8$ & $1.4 E-8$ & 0.0000 & $3.5 E-14$ \\
1.0 & 2.718281828 & 0.0000 & & 13 \\
\hline
\end{tabular}

Error: analytic-numerical.

TABLE 2: Sixth order nonlinear boundary value problem (Example 2).

\begin{tabular}{|c|c|c|c|c|c|c|}
\hline$x$ & Analytic solution & Error (HPM) & Error (VIM) & Error (ADM) & Error (ITM) & Error (DJM) \\
\hline 0.0 & 1.000000 & 0.000000 & 0.000000 & 0.000000 & 0.000000 & 0.000000 \\
\hline 0.1 & 0.904837 & $-2.4 E-7$ & $-2.4 E-7$ & $-2.4 E-7$ & $-2.4 E-7$ & $3.1 E-14$ \\
\hline 0.2 & 0.818730 & $-1.4 E-6$ & $-1.4 E-6$ & $-1.4 E-6$ & $-1.4 E-6$ & $1.9 E-13$ \\
\hline 0.3 & 0.740818 & $-3.3 E-6$ & $-3.3 E-6$ & $-3.3 E-6$ & $-3.3 E-6$ & $4.8 E-13$ \\
\hline 0.4 & 0.670320 & $-5.2 E-6$ & $-5.2 E-6$ & $-5.2 E-6$ & $-5.2 E-6$ & $8.0 E-13$ \\
\hline 0.5 & 0.606531 & $-6.2 E-6$ & $-6.2 E-6$ & $-6.2 E-6$ & $-6.2 E-6$ & $1.0 E-12$ \\
\hline 0.6 & 0.548812 & $-5.8 E-6$ & $-5.8 E-6$ & $-5.8 E-6$ & $-5.8 E-6$ & $1.0 E-12$ \\
\hline 0.7 & 0.496585 & $-4.1 E-6$ & $-4.1 E-6$ & $-4.1 E-6$ & $-4.1 E-6$ & $8.1 E-13$ \\
\hline 0.8 & 0.449329 & $-1.9 E-6$ & $-1.9 E-6$ & $-1.9 E-6$ & $-1.9 E-6$ & $4.3 E-13$ \\
\hline 0.9 & 0.406570 & $-3.6 E-7$ & $-3.6 E-7$ & $-3.6 E-7$ & $-3.6 E-7$ & $9.2 E-13$ \\
\hline 1.0 & 0.367879 & $-5.0 E-10$ & $-5.0 E-10$ & $-5.0 E-10$ & $-5.0 E-10$ & $5.6 E-14$ \\
\hline
\end{tabular}

Error: analytic-numerical.

where $f$ is a function of $x, L(u)$ is linear, and $N(u)$ is a nonlinear term. The series solution of the above functional equation is given by

$$
u=\sum_{m=0}^{\infty} u_{m} .
$$

As $L$ is linear, so we can write

$$
L\left(\sum_{m=0}^{\infty} u_{m}\right)=\sum_{m=0}^{\infty} L\left(u_{m}\right) .
$$

Let us define

$$
\begin{gathered}
G_{0}=N\left(u_{0}\right) \\
G_{k}=N\left(\sum_{m=0}^{k} u_{m}\right)-N\left(\sum_{m=0}^{k-1} u_{m}\right) .
\end{gathered}
$$

The recursive relation for the components of $u(x)$ is as follows:

$$
\begin{gathered}
u_{0}=f, \\
u_{1}=L\left(u_{0}\right)+G_{0}, \\
u_{k+1}=L\left(u_{k}\right)+G_{k}, \quad k=1,2,3, \ldots
\end{gathered}
$$

\section{Comparison of ADM and DJM}

Taylor series expansion for the nonlinear term $N(u)$ about $u_{0}$ is given by

$$
\begin{aligned}
N(u)= & N\left(u_{0}\right)+N^{\prime}\left(u_{0}\right)\left(u_{1}+u_{2}+u_{3}+\cdots\right) \\
& +\frac{N^{\prime \prime}\left(u_{0}\right)}{2 !}\left(u_{1}+u_{2}+u_{3}+\cdots\right)^{2} \\
& +\frac{N^{\prime \prime \prime}\left(u_{0}\right)}{3 !}\left(u_{1}+u_{2}+u_{3}+\cdots\right)^{3}+\cdots .
\end{aligned}
$$

In $\mathrm{ADM}$, the terms of the right hand side of the above equation are grouped to find the Adomian polynomials for the nonlinear term as follows;

$$
\begin{aligned}
\underbrace{N\left(u_{0}\right)}_{A_{0}} & +\underbrace{u_{1} N^{\prime}\left(u_{0}\right)}_{A_{1}}+\underbrace{\frac{u_{1}^{2}}{2 !} N^{\prime \prime}\left(u_{0}\right)+u_{2} N^{\prime}\left(u_{0}\right)}_{A_{2}} \\
& +\underbrace{\frac{u_{1}^{3}}{3 !} N^{\prime \prime \prime}\left(u_{0}\right)+u_{1} u_{2} N^{\prime \prime}\left(u_{0}\right)+u_{3} N^{\prime}\left(u_{0}\right)}_{A_{3}}+\cdots .
\end{aligned}
$$


TABLE 3: Sixth order nonlinear boundary value problem (Example 3).

\begin{tabular}{lcccccc}
\hline$x$ & Analytic solution & Error (ADM) & Error (VIM) & Error (ITM) & Error (HPM) & Error (DJM) \\
\hline 0.0 & 1.00000 & 0.000 & 0.000 & 0.000 & 0.000 & $-4.8 E-11$ \\
0.1 & 1.10517 & $-1.2 E-4$ & $-1.2 E-4$ & $-1.2 E-4$ & $-1.2 E-4$ & $-2.3 E-4$ \\
0.2 & 1.2214 & $-2.3 E-4$ & $-2.3 E-4$ & $-2.3 E-4$ & $-9.1 E-11$ \\
0.3 & 1.34986 & $-3.2 E-4$ & $-3.2 E-4$ & $-3.2 E-4$ & $-3.2 E-4$ & $-1.3 E-10$ \\
0.4 & 1.49182 & $-3.8 E-4$ & $-3.8 E-4$ & $-3.8 E-4$ & $-3.8 E-4$ & $-1.5 E-10$ \\
0.5 & 1.64872 & $-4.0 E-4$ & $-4.0 E-4$ & $-4.0 E-4$ & $-4.0 E-4$ & $-1.6 E-10$ \\
0.6 & 1.82212 & $-3.9 E-4$ & $-3.9 E-4$ & $-3.9 E-4$ & $-3.9 E-4$ & $-1.6 E-10$ \\
0.7 & 2.01375 & $-3.3 E-4$ & $-3.3 E-4$ & $-3.3 E-4$ & $-3.3 E-4$ & $-1.4 E-10$ \\
0.8 & 2.22554 & $-2.4 E-4$ & $-2.4 E-4$ & $-2.4 E-4$ & $-2.4 E-4$ & $-9.9 E-11$ \\
0.9 & 2.4596 & $-1.2 E-4$ & $-1.2 E-4$ & $-1.2 E-4$ & $-1.2 E-4$ & $-5.2 E-11$ \\
1.0 & 2.71828 & $+2.0 E-9$ & $+2.0 E-9$ & $+2.0 E-9$ & $+2.0 E-9$ & $+1.9 E-12$ \\
\hline
\end{tabular}

Error: analytic-numerical.

While in DJM, $N(u)$ is decomposed as follows;

$$
\begin{aligned}
\underbrace{N\left(u_{0}\right)}_{G_{0}} & +\underbrace{N\left(u_{0}+u_{1}\right)-N\left(u_{0}\right)}_{G_{1}} \\
& +\underbrace{N\left(u_{0}+u_{1}+u_{2}\right)-N\left(u_{0}+u_{1}\right)}_{G_{2}}+\cdots .
\end{aligned}
$$

Thus, in ADM and DJM, the terms of Taylor series of $N(u)$ about $u_{0}$ are grouped differently.

\section{Convergence of DJM}

The convergence of DJM is presented by Daftardar-Gejji and Bhalekar [12] as follows.

Theorem 1. If $N$ is $C^{(\infty)}$ in a neighborhood of $u_{0}$ and $\left\|N^{(n)}\left(u_{0}\right)\right\| \leq L$ for any $n$ and for some real $L>0$ and $\left\|u_{i}\right\| \leq M<1 / e, i=1,2, \ldots$, then the series $\sum_{n=0}^{\infty} G_{n}$ is absolutely convergent and moreover, $\left\|G_{n}\right\| \leq L M^{n} e^{n-1}(e-1)$, $n=1,2, \ldots$

Theorem 2. If $N$ is $C^{(\infty)}$ and $\left\|N^{(n)}\left(u_{0}\right)\right\| \leq M \leq e^{-1}$, for all $n$, then the series $\sum_{n=0}^{\infty} G_{n}$ is absolutely convergent.

\section{Analysis of DJM}

Consider a sixth order BVP of the form

$$
u^{(v i)}(x)=f(x)+g(u), \quad 0<x<b,
$$

with boundary conditions

$$
u^{i}(0)=\alpha_{i}, \quad u^{i}(b)=\beta_{i}, \quad i=0,1,2,
$$

where $f(x)$ is the source term, $g(u)$ is a continuous nonlinear function, and $\alpha_{i}, \beta_{i}$ are real finite constants. Using the operator $L=d^{(v i)} / d x^{(v i)}$, (9) becomes

$$
L u=f(x)+g(u) .
$$

Applying $L^{-1}$ (six-fold integral operator) and using the boundary conditions at $x=0$, (11) becomes

$$
\begin{aligned}
u(x)= & \alpha_{0}+\alpha_{1} x+\frac{1}{2} \alpha_{2} x^{2}+\frac{1}{3 !} A x^{3}+\frac{1}{4 !} B x^{4} \\
& +\frac{1}{5 !} C x^{5}+L^{-1}[f(x)+g(u)] .
\end{aligned}
$$

Using DJM, the solution $u(x)$ in series form is given by

$$
u(x)=\sum_{n=0}^{\infty} u_{n}(x),
$$

and the nonlinear term $g(u)$ is given by an infinite series as follows:

$$
g(u)=\sum_{n=0}^{\infty} G_{n},
$$

where $G_{n}$ 's are defined in (4). These can be constructed for various classes of nonlinearity according to the specific algorithm set by Daftardar-Gejji $[10,14]$.

Substituting (13), (14) into (12), we have

$$
\begin{aligned}
\sum_{n=0}^{\infty} u(x)= & \alpha_{0}+\alpha_{1} x+\frac{1}{2} \alpha_{2} x^{2}+\frac{1}{3 !} A x^{3}+\frac{1}{4 !} B x^{4} \\
& +\frac{1}{5 !} C x^{5}+L^{-1}[f(x)]+L^{-1}\left(\sum_{n=0}^{\infty} G_{n}\right) .
\end{aligned}
$$

DJM identifies the zeroth component $u_{0}(x)$ by all terms that arise from the boundary conditions at $x=0$ and from integrating the source term. Using DJM, the components for $u(x)$ are as follows:

$$
\begin{gathered}
u_{0}(x)=\alpha_{0}+\alpha_{1} x+\frac{1}{2} \alpha_{2} x^{2}+\frac{1}{3 !} A x^{3}+\frac{1}{4 !} B x^{4}+\frac{1}{5 !} C x^{5} \\
+L^{-1}[f(x)], \\
u_{k+1}=L^{-1}\left[G_{k}\right], \quad k \geq 0 .
\end{gathered}
$$


From here, the obtained components $u_{n}(x), n \geq 0$ of $u(x)$ contain the arbitrary constants $A, B$, and $C$. To determine these constants, we impose the boundary conditions at $x=b$ to the approximant $\Phi_{k}=\sum_{k=0}^{n-1} u_{k}$. The resulting algebraic system of equations can be solved to determine the values of these constants for the series solution of the sixth order BVP.

\section{Numerical Results}

In this section, we solve three nonlinear boundary value problems by using DJM. Fifth order nonlinear BVP is solved initially and the rest of the two examples are of sixth order.

Example 1. Consider the fifth order BVP

$$
u^{(5)}(x)=e^{-x} u^{2}(x), \quad 0<x<1,
$$

subject to the boundary conditions

$$
u(0)=u^{\prime}(0)=u^{\prime \prime}(0)=1, \quad u(1)=u^{\prime}(1)=e .
$$

The theoretical solution for this problem is

$$
u(x)=e^{x}
$$

Writing (17) in an operator form, we have

$$
L u=e^{-x} u^{2}(x)
$$

Applying $L^{-1}$ (five-fold integral operator) to (20) and using the boundary conditions at $x=0$, we can write

$$
u(x)=1+x+\frac{1}{2} x^{2}+\frac{1}{6} A x^{3}+\frac{1}{24} B x^{4}+L^{-1}\left(e^{-x} u^{2}(x)\right)
$$

The constants $A, B$ are to be determined. Now using DJM, replacing $u(x)$ and $u^{2}(x)$ by the series defined in (13) and (14), we get

$$
\begin{aligned}
\sum_{n=0}^{\infty} u_{n}(x)= & +x+\frac{1}{2} x^{2}+\frac{1}{6} A x^{3}+\frac{1}{24} B x^{4} \\
& +L^{-1}\left(e^{-x} \sum_{n=0}^{\infty} G_{n}\right) .
\end{aligned}
$$

The components of $u(x)$ can be found using (16) as follows;

$$
\begin{gathered}
u_{0}=1+x+\frac{1}{2} x^{2}+\frac{1}{6} A x^{3}+\frac{1}{24} B x^{4}, \\
u_{k+1}=L^{-1}\left(e^{-x} G_{k}\right), \quad k \geq 0,
\end{gathered}
$$

where $G_{n}$ 's are defined in (4). The components of $u(x)$ are given as

$$
\begin{aligned}
& u_{1}=L^{-1}\left(e^{-x} G_{0}\right) \\
& =\frac{x^{5}}{120}+\frac{x^{6}}{720}+\frac{x^{7}}{5040}+\left(-\frac{1}{40320}+\frac{A}{20160}\right) x^{8} \\
& +\left(-\frac{1}{362880}+\frac{B}{181440}\right) x^{9} \\
& -\frac{x^{10}}{3628800}+\frac{\left(19-40 A+20 A^{2}\right) x^{11}}{39916800} \\
& +\frac{\left(-71+210 A-140 A^{2}-70 B+70 A B\right) x^{12}}{479001600} \\
& +\frac{\left(181-672 A+560 A^{2}+420 B-560 A B+70 B^{2}\right) x^{13}}{6227020800} \\
& +\left(\left(-379+1680 A-1680 A^{2}-1512 B+2520 A B\right.\right. \\
& \left.\left.-630 B^{2}\right) x^{14}\right) \times(87178291200)^{-1}+O\left(x^{15}\right) \\
& u_{2}=L^{-1}\left(e^{-x} G_{1}\right) \\
& =\frac{x^{10}}{1814400}+\frac{x^{11}}{19958400}+\frac{x^{12}}{239500800} \\
& +\left(-\frac{19}{1037836800}+\frac{29 A}{1556755200}\right) x^{13} \\
& +\frac{(293-420 A+128 B) x^{14}}{43589145600}+O\left(x^{15}\right)
\end{aligned}
$$

and so on. The required solution in series form is

$$
\begin{aligned}
u= & u_{0}+u_{1}+u_{2} \\
= & 1+x+\frac{x^{2}}{2}+\frac{A x^{3}}{6}+\frac{B x^{4}}{24}+\frac{x^{5}}{120}+\frac{x^{6}}{720}+\frac{x^{7}}{5040} \\
& +\frac{(-1+2 A) x^{8}}{40320}+\frac{(-1+2 B) x^{9}}{362880} \\
& +\frac{x^{10}}{3628800}+\frac{\left(21-40 A+20 A^{2}\right) x^{11}}{39916800} \\
& -\frac{\left(69-210 A+140 A^{2}+70 B-70 A B\right) x^{12}}{479001600} \\
& +\frac{\left(67-556 A+560 A^{2}+420 B-560 A B+70 B^{2}\right) x^{13}}{6227020800} \\
& -\left(\left(-207-840 A+1680 A^{2}+1256 B\right.\right. \\
& \times(87178291200)^{-1}+O\left(x^{15}\right) .
\end{aligned}
$$


To find the values of unknown constants $A$ and $B$, we impose the boundary conditions at $x=1$ on the three-term approximant $\Phi_{n}=\sum_{n=0}^{2} u_{n}$ to obtain the following system of equations;

$$
\begin{aligned}
& \frac{519070837 A}{3113510400}+\frac{29 A^{2}}{103783680}+\frac{908225291 B}{21794572800} \\
& +\frac{53 A B}{622702080}+\frac{B^{2}}{249080832}=e-\frac{8752329923}{3487131648} \\
& \frac{778984793 A}{1556755200}+\frac{113 A^{2}}{38918880}+\frac{259534741 B}{1556755200} \\
& +\frac{A B}{1010880}+\frac{B^{2}}{22239360}=e-\frac{182467069}{88957440} .
\end{aligned}
$$

Solving this system of equations, we get

$$
A=0.9999999999456785, \quad B=1.0000000002360663 \text {. }
$$

Therefore the numerical solution is given by

$$
\begin{aligned}
u(x)= & 1+x+\frac{x^{2}}{2}+0.166667 x^{3}+0.0416667 x^{4} \\
& +\frac{x^{5}}{120}+\frac{x^{6}}{720}+\frac{x^{7}}{5040}+0.0000248016 x^{8} \\
& +2.75573192369966 \times 10^{-6} x^{9}+\frac{x^{10}}{3628800} \\
& +2.505210838544172 \times 10^{-8} x^{11} \\
& +2.087675698786751 \times 10^{-9} x^{12} \\
& +1.6059043833333186 \times 10^{-10} x^{13} \\
& +1.1470745608558747 \times 10^{-11} x^{14} .
\end{aligned}
$$

Example 2. We next consider the sixth order nonlinear BVP as

$$
u^{v i}(x)=e^{x} u^{2}(x), \quad 0<x<1,
$$

subject to the boundary conditions

$$
\begin{aligned}
& u(0)=1, \quad u^{\prime}(0)=-1, \quad u^{\prime \prime}(0)=1, \\
& u(1)=e^{-1}, \quad u^{\prime}(1)=-e^{-1}, \quad u^{\prime \prime}(1)=e^{-1},
\end{aligned}
$$

the theoretical solution for this problem is

$$
u(x)=e^{-x} \text {. }
$$

Using the operator $L=d^{v i} / d x^{v i}$, (29) can be written as

$$
L u=e^{x} u^{2}(x) \text {. }
$$

Operating the last equation with $L^{-1}$ and using the boundary conditions at $x=0$, we can write

$$
\begin{aligned}
u(x)= & 1-x+\frac{1}{2} x^{2}+\frac{1}{6} A x^{3}+\frac{1}{24} B x^{4}+\frac{1}{120} C x^{5} \\
& +L^{-1}\left[e^{x} u^{2}(x)\right]
\end{aligned}
$$

where the inverse operator $L^{-1}$ is a six-fold integral from 0 to $x$ and $A, B, C$ are constants to be determined in a parallel manner to our discussion above. Substituting the series for $u(x)$ and $u^{2}(x)$ in (33), we have

$$
\begin{aligned}
\sum_{n=0}^{\infty} u_{n}(x)= & 1-x+\frac{1}{2} x^{2}+\frac{1}{6} A x^{3}+\frac{1}{24} B x^{4}+\frac{1}{120} C x^{5} \\
& +L^{-1}\left[e^{x} \sum_{n=0}^{\infty} G_{n}\right] .
\end{aligned}
$$

To determine the components $u_{n}(x), n \geq 1$, we use DJM as follows:

$$
\begin{gathered}
u_{0}(x)=1-x+\frac{1}{2} x^{2}+\frac{1}{6} A x^{3}+\frac{1}{24} B x^{4}+\frac{1}{120} C x^{5} \\
u_{k+1}=L^{-1}\left[e^{x} G_{k}\right], \quad k \geq 1
\end{gathered}
$$

where $G_{n}$ 's are defined in (4) and (9). Therefore the required components are

$$
\begin{aligned}
& u_{0}(x)=1-x+\frac{1}{2} x^{2}+\frac{1}{6} A x^{3}+\frac{1}{24} B x^{4}+\frac{1}{120} C x^{5} \\
& u_{1}(x) \\
& =L^{-1}\left[e^{x} G_{0}\right] \\
& =\frac{x^{6}}{720}-\frac{x^{7}}{5040}+\frac{x^{8}}{40320}+\left(\frac{1}{362880}+\frac{A}{181440}\right) x^{9} \\
& +\left(-\frac{1}{3628800}+\frac{B}{1814400}\right) x^{10} \\
& +\left(\frac{1}{39916800}+\frac{G}{19958400}\right) x^{11} \\
& +\frac{\left(19+40 A+20 A^{2}\right) x^{12}}{479001600} \\
& +\frac{\left(71+210 A+140 A^{2}+70 B+70 A B\right) x^{13}}{6227020800} \\
& +\left(\left(181+672 A+560 A^{2}+420 B+560 A B\right.\right. \\
& \left.\left.+70 B^{2}+112 G+112 A G\right) x^{14}\right) \\
& +(x)=L^{-1}\left[e^{x} G_{1}\right] \\
& +x^{12} \\
& +(839500800 \\
& +\frac{x^{13}}{3113510400}
\end{aligned}
$$


So the required solution is

$$
\begin{aligned}
u(x) & u_{0}+u_{1}+u_{2} \\
= & 1-x+\frac{x^{2}}{2}+\frac{A x^{3}}{6}+\frac{B x^{4}}{24}+\frac{G x^{5}}{120}+\frac{x^{6}}{720}-\frac{x^{7}}{5040} \\
& +\frac{x^{8}}{40320}+\frac{(1+2 A) x^{9}}{362880}+\frac{(-1+2 B) x^{10}}{3628800} \\
& +\frac{(1+2 G) x^{11}}{39916800}+\frac{\left(21+40 A+20 A^{2}\right) x^{12}}{479001600} \\
& +\frac{\left(69+210 A+140 A^{2}+70 B+70 A B\right) x^{13}}{6227020800} \\
& +\left(\left(183+672 A+560 A^{2}+420 B+560 A B\right.\right. \\
& \times(87178291200)^{-1} .
\end{aligned}
$$

Using the boundary conditions at $x=1$, we have

$$
\begin{gathered}
\frac{74133707 A}{444787200}+\frac{A^{2}}{14152320}+\frac{64865683 B}{1556755200}+\frac{A B}{56609280} \\
+\frac{B^{2}}{1245404160}+\frac{162163 G}{19459440}+\frac{A G}{778377600} \\
=e^{-1}-\frac{539448331}{1076275200} \\
\frac{518971487 A}{1037836800}+\frac{5 A^{2}}{5660928}+\frac{20757449 B}{124540416}+\frac{7 A B}{29652480} \\
+\frac{B^{2}}{88957440}+\frac{32432843 G}{778377600}+\frac{A G}{55598400} \\
=-e^{-1}-\frac{56341}{7862400} \\
\frac{19966673 A}{19958400}+\frac{61 A^{2}}{5987520}+\frac{1330699 B}{2661120}+\frac{A B}{342144} \\
=e^{-1}-\frac{874289}{844800} . \\
+\frac{1247443 G}{7484400}+\frac{A G}{4276800}
\end{gathered}
$$

Solving this system of equations for $A, B$, and $G$, we get

$$
\begin{gathered}
A=-1.0000000002343303, \\
B=1.0000000020461832, \\
G=-1.0000000056306735 .
\end{gathered}
$$

So the required series solution is given by

$$
\begin{aligned}
u(x)= & 1-x+\frac{x^{2}}{2}-0.166667 x^{3}+0.0416667 x^{4} \\
& -0.00833333 x^{5}+\frac{x^{6}}{720}-\frac{x^{7}}{5040}+\frac{x^{8}}{40320} \\
& -2.7557319236900926 \times 10^{-6} x^{9} \\
& +2.755731933676053 \times 10^{-7} x^{10} \\
& -2.5052108667562203 \times 10^{-8} x^{11} \\
& +2.0876756987868175 \times 10^{-9} x^{12} \\
& -1.605904383682184 \times 10^{-10} x^{13} \\
& +1.1470745597729725 \times 10^{-11} x^{14}+O\left(x^{15}\right) .
\end{aligned}
$$

In the following example, we consider the BVP in which the boundary conditions are given at the even-order derivatives. The assumption that the boundary conditions be taken at the even-order derivatives is in fact used by Boutayeb and Twizell $[15,16]$.

Example 3. Let us take the nonlinear BVP of the form

$$
u^{(v i)}=e^{-x} u^{2}(x), \quad 0<x<1,
$$

subject to the boundary conditions

$$
\begin{aligned}
& u(0)=u^{\prime \prime}(0)=u^{(i v)}(0)=1, \\
& u(1)=u^{\prime \prime}(1)=u^{(i v)}(1)=e .
\end{aligned}
$$

The exact solution of this problem is $u=e^{x}$.

Equation (41) can be written in an operator form as

$$
L u=e^{-x} u^{2}(x)
$$

Applying $L^{-1}$ (six-fold integral operator) to (43) and using the boundary conditions at $x=0$ yields

$$
\begin{aligned}
u(x)= & +A x+\frac{1}{2} x^{2}+\frac{1}{6} B x^{3}+\frac{1}{24} x^{4}+\frac{1}{120} C x^{5} \\
& +L^{-1}\left[e^{-x} u^{2}(x)\right]
\end{aligned}
$$

where $A, B$, and $C$ are constants to be determined by using the boundary conditions at $x=1$. Substituting the series for $u(x)$ and for the nonlinear term $u^{2}(x)$ in (44), we have

$$
\begin{aligned}
\sum_{n=0}^{\infty} u_{n}(x)= & 1+A x+\frac{1}{2} x^{2}+\frac{1}{6} B x^{3}+\frac{1}{24} x^{4}+\frac{1}{120} C x^{5} \\
& +L^{-1}\left(e^{-x} \sum_{n=0}^{\infty} G_{n}\right) .
\end{aligned}
$$


Following the same criteria as in Example 2, we conclude that

$$
\begin{aligned}
& u(x)=1+A x+\frac{x^{2}}{2}+\frac{B x^{3}}{6}+\frac{x^{4}}{24}+\frac{G x^{5}}{120}+\frac{x^{6}}{720} \\
& +\frac{(-1+2 A) x^{7}}{5040}+\frac{\left(3-4 A+2 A^{2}\right) x^{8}}{40320} \\
& -\frac{\left(7-12 A+6 A^{2}-2 B\right) x^{9}}{362880} \\
& +\frac{\left(21-32 A+12 A^{2}-8 B+8 A B\right) x^{10}}{3628800} \\
& -\frac{\left(61-80 A+20 A^{2}-40 B+40 A B-2 G\right) x^{11}}{39916800} \\
& +\left(\left(183-192 A+30 A^{2}-160 B+120 A B+20 B^{2}\right.\right. \\
& \left.-12 G+12 A G) x^{12}\right) \times(479001600)^{-1} \\
& -\left(\left(549-452 A+42 A^{2}-560 B+280 A B+140 B^{2}\right.\right. \\
& \left.-84 G+84 A G) x^{13}\right) \times(6227020800)^{-1} \\
& +\left(\left(1661-1080 A+92 A^{2}-1792 B+560 A B\right.\right. \\
& \left.\left.+560 B^{2}-448 G+336 A G+112 B G\right) x^{14}\right) \\
& \times(87178291200)^{-1}+O\left(x^{15}\right) .
\end{aligned}
$$

In order to find the values of $A, B$, and $G$, we impose the boundary conditions at $x=1$ on the three-term approximant and then solve the obtained algebraic system of equations to get

$$
\begin{gathered}
A=1.00000000048, \quad B=0.9999999958, \\
G=1.000000025
\end{gathered}
$$

for the solution which is

$$
\begin{aligned}
u(x)= & +1 . x+\frac{x^{2}}{2}+0.166667 x^{3}+\frac{x^{4}}{24} \\
& +0.00833333 x^{5}+\frac{x^{6}}{720}+0.000198413 x^{7} \\
& +0.0000248016 x^{8}+2.7557318995 \times 10^{-6} x^{9} \\
& +2.7557319224 \times 10^{-7} x^{10} \\
& +2.5052109648 \times 10^{-8} x^{11}+2.0876756988 \\
& \times 10^{-9} x^{12}+1.6059043867 \times 10^{-10} x^{13} \\
& +1.1470745597 \times 10^{-11} x^{14} .
\end{aligned}
$$

\section{Concluding Remarks}

The computations related with the three examples discussed above were performed by using Mathematica 7.0. We therefore conclude that the proposed algorithm (DJM) produced a rapidly convergent series as compared to modified ADM, VIM, ITM, and HPM. The other works, for example, spline solution, required restrictions on the boundary conditions and the ADM needs tedious calculations to find the Adomian polynomials, whereas no restrictions or complicated calculations are required in this approach. Moreover, numerical methods based on DJM would require less computational effort.

\section{Conflict of Interests}

The authors declare that there is no conflict of interests regarding the publication of this paper.

\section{References}

[1] A. R. Davies, A. Karageorghis, and T. N. Phillips, "Spectral Galerkin methods for the primary two-point boundary value problem in modelling viscoelastic flows," International Journal for Numerical Methods in Engineering, vol. 26, no. 3, pp. 647662, 1988.

[2] A. R. Davies, A. Karageorghis, and T. N. Phillips, "Spectral collocation methods for the primary two-point boundary value problem in modelling viscoelastic flows," International Journal for Numerical Methods in Engineering, vol. 26, no. 4, pp. 805813, 1988.

[3] R. P. Agarwal, Boundary Value Problems for High Ordinary Differential Equations, World Scientific, Singapore, 1986.

[4] M. S. Khan, Finite difference solutions of fifth order boundary value problems [Ph.D. thesis], Brunel University, London, UK, 1994.

[5] H. N. Caglar, S. H. Caglar, and E. H. Twizell, “The numerical solution of fifth-order boundary value problems with sixthdegree B-spline functions," Applied Mathematics Letters, vol. 12, no. 5, pp. 25-30, 1999.

[6] A.-M. Wazwaz, "The numerical solution of fifth-order boundary value problems by the decomposition method," Journal of Computational and Applied Mathematics, vol. 136, no. 1-2, pp. 259-270, 2001.

[7] E. H. Twizell and A. Boutayeb, "Numerical methods for the solution of special and general sixth-order boundary value problems, with applications to Bénard layer eigenvalue problems," Proceedings of the Royal Society of London A, vol. 431, no. 1883, pp. 433-450, 1990.

[8] P. Baldwin, "Asymptotic estimates of the eigenvalues of a sixth-order boundary-value problem obtained by using global phase-integral methods," Philosophical Transactions of the Royal Society of London A, vol. 322, no. 1566, pp. 281-305, 1987.

[9] P. Baldwin, "Localised instability in a Bénard layer," Applicable Analysis, vol. 24, no. 1-2, pp. 117-156, 1987.

[10] M. M. Chawla and C. P. Katti, "Finite difference methods for two-point boundary value problems involving high order differential equations," BIT Numerical Mathematics, vol. 19, no. 1, pp. 27-33, 1979.

[11] A.-M. Wazwaz, "The numerical solution of sixth-order boundary value problems by the modified decomposition method," 
Applied Mathematics and Computation, vol. 118, no. 2-3, pp. 311325, 2001.

[12] V. Daftardar-Gejji and S. Bhalekar, "Solving fractional boundary value problems with Dirichlet boundary conditions using a new iterative method," Computers \& Mathematics with Applications, vol. 59, no. 5, pp. 1801-1809, 2010.

[13] I. Ullah, H. Khan, and M. T. Rahim, "Numerical solutions of higher order nonlinear boundary value problems by new iterative method," Applied Mathematical Sciences, vol. 7, no. 49, pp. 2429-2439, 2013.

[14] V. Daftardar-Gejji and H. Jafari, "An iterative method for solving nonlinear functional equations," Journal of Mathematical Analysis and Applications, vol. 316, no. 2, pp. 753-763, 2006.

[15] J. Toomore, J. R. Zahn, J. Latour, and E. A. Spiegel, "Stellar convection theory-II: single-mode study of the second convection zone in an A-type star," Astrophysical Journal, vol. 207, part 1, pp. 545-563, 1976.

[16] A. Boutayeb and E. H. Twizell, "Numerical methods for the solution of special sixth-order boundary-value problems," International Journal of Computer Mathematics, vol. 45, no. 3-4, pp. 207-233, 1992. 

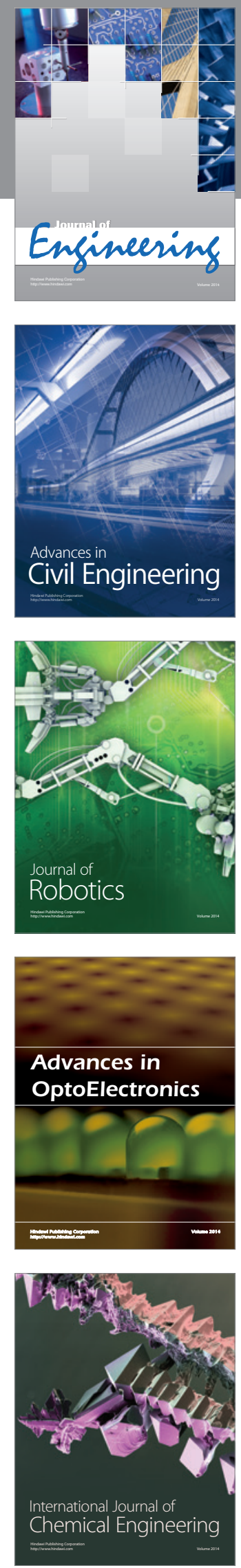

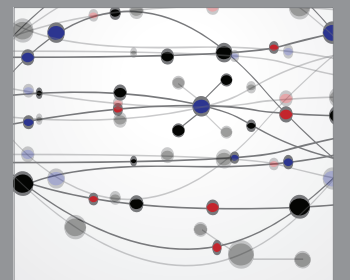

The Scientific World Journal
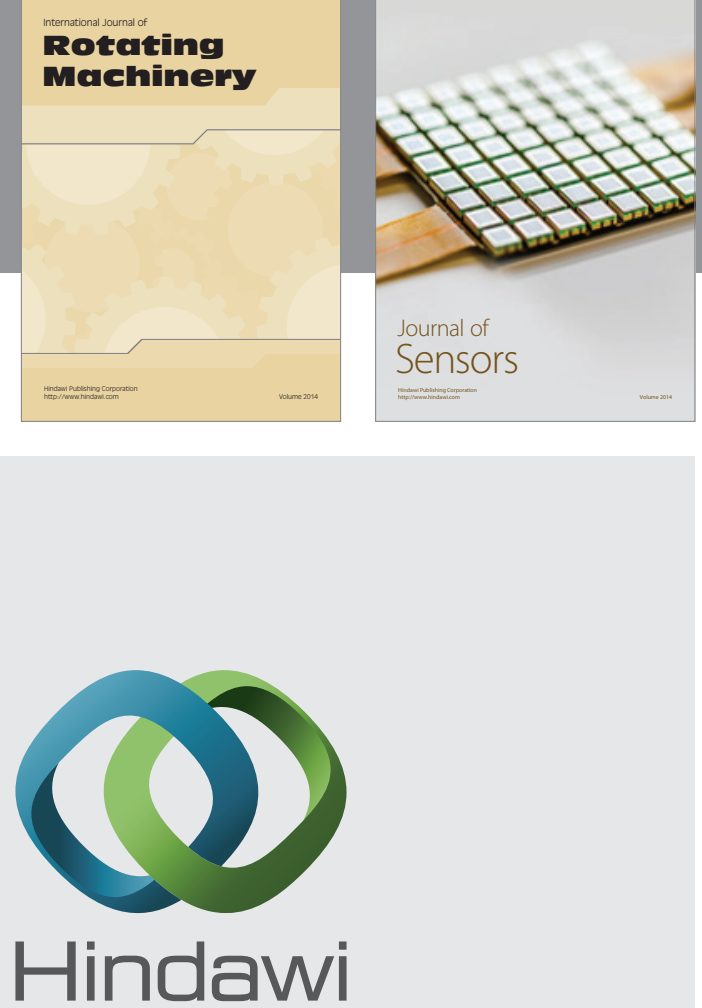

Submit your manuscripts at http://www.hindawi.com
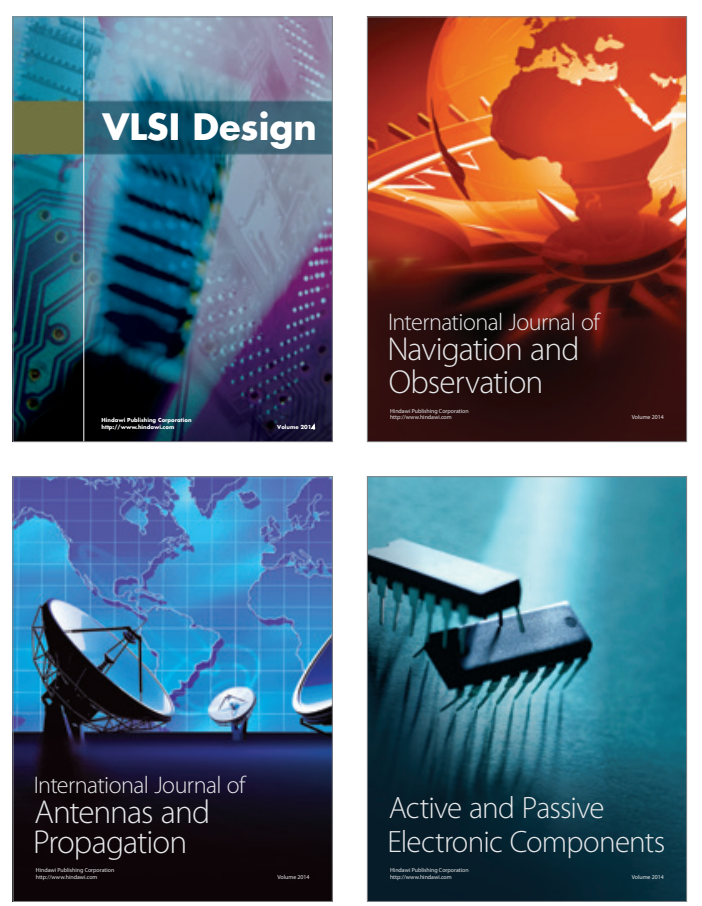
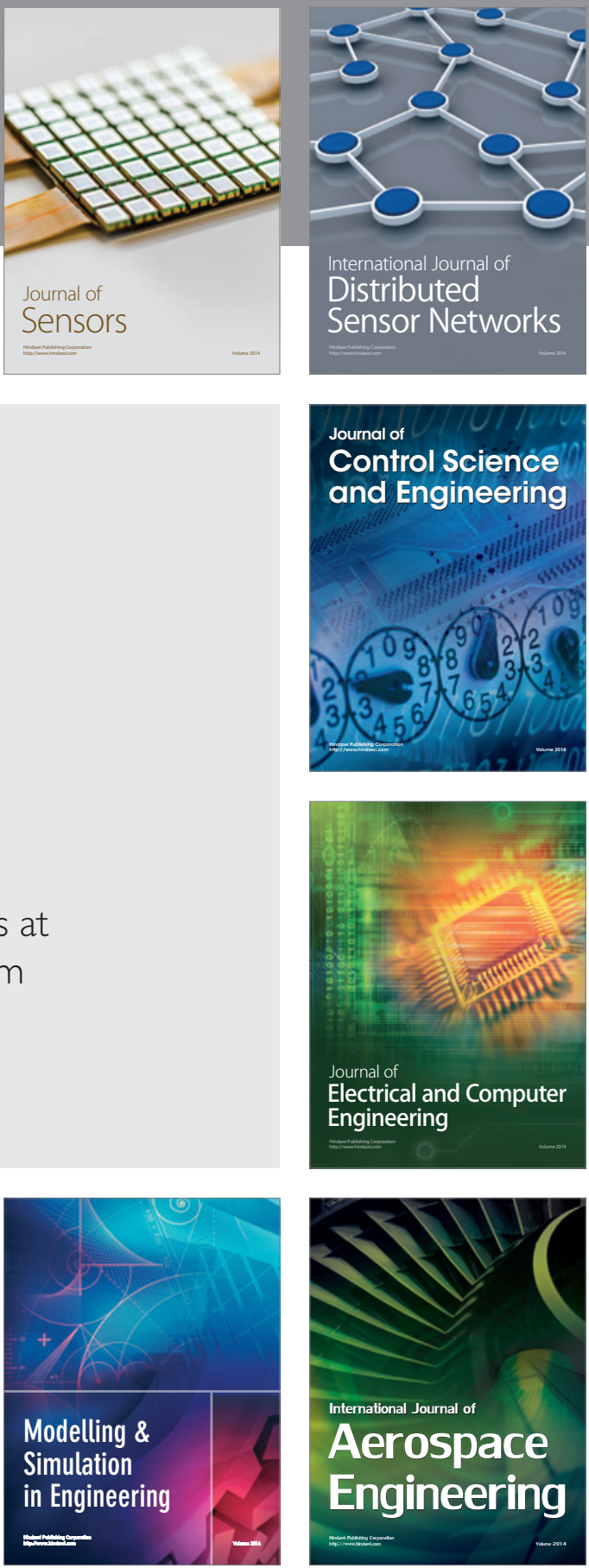

Journal of

Control Science

and Engineering
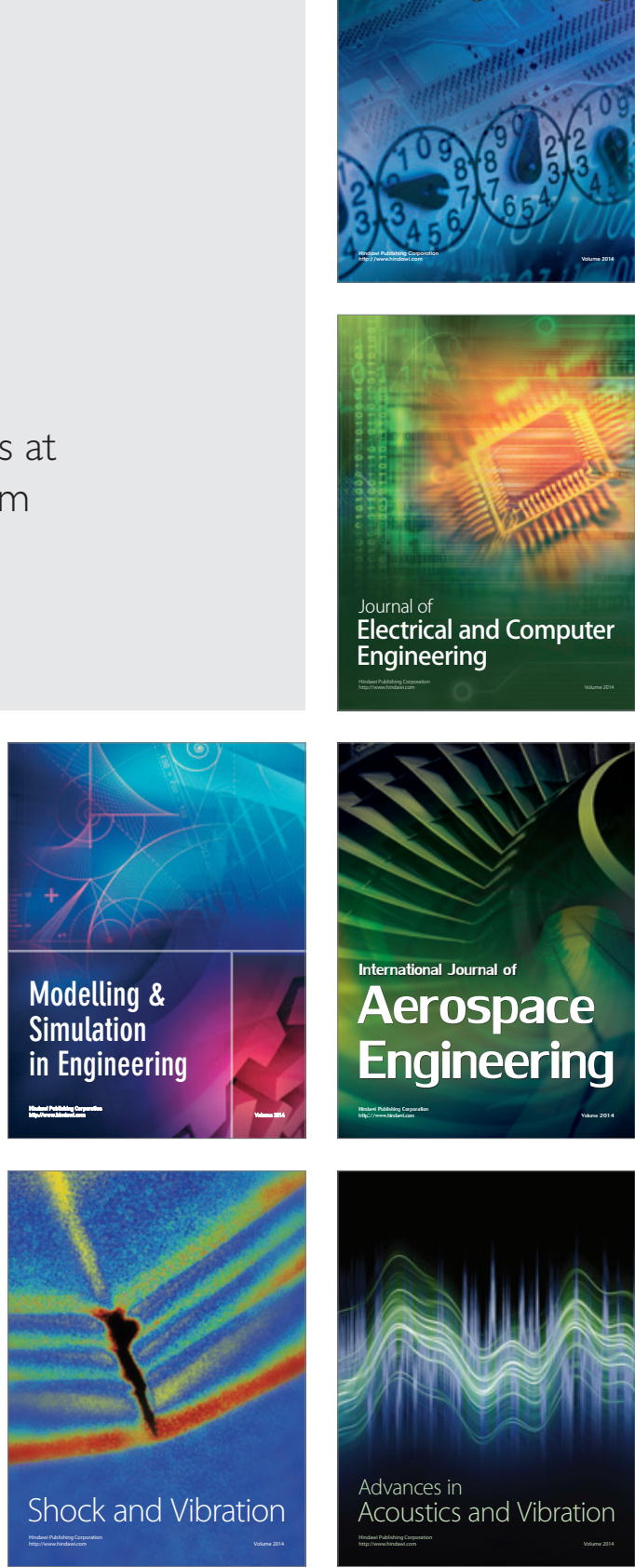